\title{
Coordinated Multi-Point Decoding with Dual-polarized Antennas
}

\author{
Symeon Chatzinotas*, Dimitrios Christopoulos*, Björn Ottersten*† \\ ${ }^{*}$ SnT - securityandtrust.lu, University of Luxembourg, \\ Email: \{Symeon.Chatzinotas, Dimitrios.Christopoulos, Bjorn.Ottersten\}@uni.lu \\ ${ }^{\dagger}$ Royal Institute of Technology (KTH), Sweden, Email: bjorn.ottersten@ee.kth.se
}

\begin{abstract}
Coordinated multi-point processing has shown great potential for cellular networks, while multiple antenna systems (MIMO) is the key to next generation wireless communications. Full exploitation of MIMO technology, however, demands high antenna separation at the transceivers. This paper investigates the use of dual polarized antennas as a mean to overcome hardware size limitations. Uplink ergodic sum-rate capacity of a multicell joint processing (MJP) system employing dual polarized antennas is evaluated through theoretical analysis. Results are supported by numerical simulations. The designed system incorporates uniformly distributed users, path loss and Rayleigh fading, thus extending the well known Wyner model. Optimal and MMSE receiver architectures are compared in terms of capacity and complexity. System capacity is calculated with respect to cell size or cross polar discrimination (XPD). The results support the use of dual-polar decoding for low XPD, dense cellular systems while per polarization processing is acceptable in high XPD, sparse systems.
\end{abstract}

Index Terms-CoMP, Multicell Joint Decoding, Dual Polarization, Optimal/MMSE Capacity.

\section{INTRODUCTION}

According to [1], in order to avoid antenna correlation a separation of half wavelength is needed at the mobile terminals, while a separation of ten wavelengths at the Base Station (BS). Furthermore, MIMO capacity has been shown to scale linearly with the minimum number of transmit and receive antennas [2] and thus it would be desirable to incorporate a large number of antennas in future transceivers. This is not always feasible due to space limitations at mobile terminals or large wavelengths (e.g. in UHF) and this is where dualpolarized antennas come to play. Originally, dual-polarization has been utilized as a means of creating two parallel lowinterference channels, which could be exploited for diversity gain or interference mitigation. In this paper, dual polarization will be utilized for spatial multiplexing and various multiuser receive architectures will be compared in terms of performance and complexity.

\section{A. Notation}

Throughout the formulations of this paper, $\mathbb{E}[\cdot]$ denotes the expectation, $(\cdot)^{H}$ denotes the conjugate transpose matrix, $(\cdot)^{T}$ denotes the transpose matrix, $\odot$ denotes the Hadamard product and $\otimes$ denotes the Kronecker product.The Frobenius norm of a matrix or vector is denoted by $\|\cdot\| . \mathbf{I}_{n}$ denotes a $n \times n$ identity matrix, $\mathbb{I}_{n \times m}$ a $n \times m$ matrix of ones, $\mathbf{1}_{n}$ a $n \times 1$ vector of ones, $\mathbf{0}$ a zero matrix and $\mathbf{G}_{n \times m}$ a $n \times m$ Gaussian matrix. The figure of merit analyzed and compared throughout this paper is the ergodic per-cell sum-rate capacity.

\section{Preliminaries \& Related Work}

\section{A. Coordinated Multi-Point}

Coordinated Multi-Point (CoMP) transmission/reception is a term employed by 3GPP LTE-A standardization initiative in order to describe a group of advanced multi-cell coordination techniques [3]. In this paper, we focus on Multicell Joint Processing (MJP) in the uplink channel, where user signals received by multiple BSs are jointly decoded in order to mitigate inter-cell interference. This MJP scenario has appeared in the literature under various research topics, such as BS cooperation, Network MIMO and Distributed Antenna Systems (DAS). Its sum-rate capacity has been studied for optimal and MMSE receivers [4], as well as for various channel impairments, such as path loss [5], shadowing [6], fading [7] and antenna correlation [4]. In this paper, we consider both optimal and MMSE receivers for an MJD model incorporating spatially distributed users, path loss, Rayleigh fading and dual polarized antennas.

\section{B. Polarization}

Polarization of electromagnetic signals is achieved by specialized antennas which can shape the vectors of the electric and magnetic filed. Various polarizations schemes have been proposed and employed, such as horizontal/vertical/slant polarization (commonly for terrestrial systems), right/left hand side circular polarization (mostly for satellite systems) and even 3D polarization [8]. With the advent of multiple antenna systems, polarization has been treated as an additional degree of freedom which can be used for diversity or multiplexing gains [9], [10] . In this context, the dilemma that naturally rises is co-polar vs. cross-polar antennas. In general, replacing two co-polar antennas with two cross-polar antennas comes with a cost and the optimum configuration strongly depends on the antenna and channel characteristics. In the former case, the communication performance is limited by the antenna correlation due to the close proximity of the two co-polar antennas, while in the latter case by the power imbalance due to the XPD of the cross-polar antennas [11], [12]. 


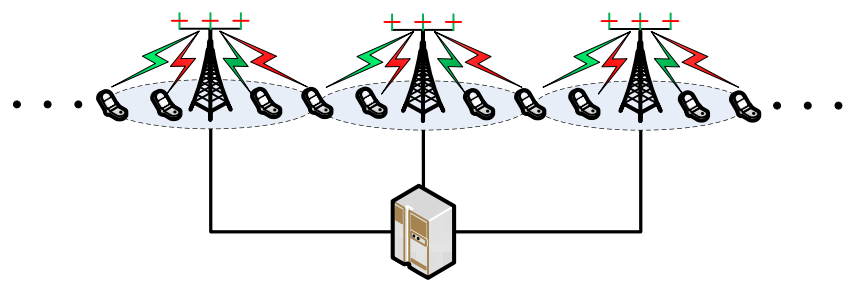

Fig. 1. Coordinated multi-point processing system with dual polarized antennas.

\section{IND Channel}

The Independent Non-identically Distributed (IND) channel [13] is a generalization of the Gaussian multi-antenna channel with independent identically distributed (i.i.d.) complex circularly symmetric (c.c.s.) entries which is commonly used in single- user MIMO research. More specifically, the IND channel is a multidimensional channel that includes independent complex circularly symmetric elements with different variances. In the literature, it has been utilized to describe a range of channel impairments, such as multiuser path-loss models [5], polarization [14] and separable correlation [4], [15].

\section{Channel Model}

Let us consider an ideal MJD system comprised by $N$ BSs each equipped with $M$ dual-polarized antenna pairs (i.e. $2 \times M$ antennas). All BSs are interconnected to a central processor who is responsible for jointly decoding the received signals. Each User Terminal (UT) is equipped with one dual-polarized antenna pair (i.e. two antennas ), while the horizontally and vertically polarized antenna components are assumed to be uncorrelated. In addition, $K$ UTs are considered to be spatially distributed across the system's coverage area. Firstly, let us consider the channel between a BS antenna and a UT,

$$
\mathbf{H}_{\mathrm{p}}=\left[\begin{array}{ll}
h_{00} & h_{01} \\
h_{10} & h_{11}
\end{array}\right]=\mathbf{X} \odot \mathbf{G},
$$

where $\mathbb{E}\left[\left|h_{00}\right|^{2}\right]=E\left[\left|h_{11}\right|^{2}\right]=1$ and $E\left[\left|h_{01}\right|^{2}\right]=E\left[\left|h_{10}\right|^{2}\right]=$ $\sqrt{\chi}, \mathbf{G} \sim \mathcal{C N}\left(\mathbf{0}, \mathbf{I}_{2}\right)$ and

$$
\mathbf{X}=\left[\begin{array}{cc}
1 & \sqrt{\chi} \\
\sqrt{\chi} & 1
\end{array}\right]
$$

The variable $\chi$ varies in [0,1] and quantifies the inverse of the cross polar discrimination (XPD), where $0 \leq X P D<\infty$. Now let us assume the MIMO Multiple Access Channel (MAC) which comprises $N K$ transmitting UTs and $N M$ receiving BS antennas. This can be written as the vectorial form of a discrete memoryless channel

$$
\mathbf{y}=\mathbf{H x}+\mathbf{z},
$$

where $\mathbf{x}$ is a $2 N K \times 1$ vector containing the transmit Gaussian symbols, $\mathbf{y}$ is a $2 N M \times 1$ vector containing the received symbols and $\mathbf{z} \sim \mathcal{C N}(\mathbf{0}, \mathbf{I})$ is a $2 N M \times 1$ vector representing
AWGN. The system channel matrix $\mathbf{H}$ can be written in more detail as:

$$
\begin{aligned}
\mathbf{H} & =\mathbf{\Omega} \odot \mathbf{G} \\
\text { with } \quad \boldsymbol{\Omega} & =\left(\boldsymbol{\Sigma} \otimes \mathbb{I}_{2}\right) \odot\left(\mathbb{I}_{N M \times N K} \otimes \mathbf{X}\right),
\end{aligned}
$$

where $\boldsymbol{\Sigma}$ is a $N M \times N K$ positive definite matrix containing the path loss coefficients for all $N^{2} M K$ BS-UT links. In cases where the two polarizations are treated independently, the copolar and cross-polar received signals can be expressed as:

$$
\begin{aligned}
\mathbf{H}_{00}=\boldsymbol{\Sigma} \odot \mathbf{G}_{00} & \mathbf{H}_{01}=\sqrt{\chi} \boldsymbol{\Sigma} \odot \mathbf{G}_{01} \\
\mathbf{H}_{10}=\sqrt{\chi} \boldsymbol{\Sigma} \odot \mathbf{G}_{10} & \mathbf{H}_{11}=\boldsymbol{\Sigma} \odot \mathbf{G}_{11}
\end{aligned}
$$

where $\mathbf{G}_{i j}, \forall i, j \in\{0,1\}$ are independent Gaussian submatrices of $\mathbf{G}$. For $i=0$ only odd rows are selected, while for $i=1$ only even. Columns are selected accordingly based on the value of $j$. Similarly, we define the following submatrices:

$$
\begin{aligned}
& \mathbf{H}_{0}=\left(\boldsymbol{\Sigma} \otimes \mathbf{1}_{2}^{T}\right) \odot\left(\mathbb{I}_{N M \times N K} \otimes[1 \sqrt{\chi}]\right) \odot \mathbf{G}_{0}, \\
& \mathbf{H}_{1}=\left(\boldsymbol{\Sigma} \otimes \mathbf{1}_{2}^{T}\right) \odot\left(\mathbb{I}_{N M \times N K} \otimes[\sqrt{\chi} 1]\right) \odot \mathbf{G}_{1},
\end{aligned}
$$

for odd and even rows respectively.

\section{RECEIVER ARCHITECTURES}

This section investigates a range of receive architectures in terms of ergodic sum-rate capacity and complexity.

\section{A. Optimal Joint Decoder}

In this receiver architecture, all the user signals received in both polarizations are jointly decoded using Successive Interference Cancellation (SIC). This is the optimal receiver which achieves the channel capacity as shown in [16]. In this context, the ergodic sum-rate capacity normalized by the number of cells is given by:

$$
\mathrm{C}_{1}=\frac{1}{N} \log \operatorname{det}\left(\mathbf{I}_{2 N M}+\gamma \mathbf{H} \mathbf{H}^{H}\right),
$$

where $\gamma$ denotes the transmit $\mathrm{SNR}^{1}$ per polarization for each UT.

\section{B. MMSE Linear Filtering}

In this case, single-user decoding is employed after MMSE linear filtering of both polarizations. As shown in [4], the ergodic sum-rate capacity normalized by the number of cells is bounded by:

$$
\mathrm{C}_{2} \geq-2 K \log \left(\frac{1}{2 N K} \operatorname{tr}\left[\left(\mathbf{I}_{2 N K}+\gamma \mathbf{H}^{H} \mathbf{H}\right)^{-1}\right]\right) .
$$

\footnotetext{
${ }^{1}$ Transmit SNR is defined as the transmit power normalized by the receiver noise.
} 


\section{Optimal Joint Decoder per Polarization}

Now the signals received in each individual polarization are optimally decoded considering the other polarization as interference. It should be noted that CSI is available for both polarizations. The ergodic sum-rate capacity normalized by the number of cells is given by:

$$
\begin{aligned}
\mathrm{C}_{3} & =\frac{1}{N} \log \operatorname{det}\left(\mathbf{I}_{N M}+\gamma \mathbf{H}_{00} \mathbf{H}_{00}^{H} \mathbf{R}_{01}^{-1}\right) \\
& +\frac{1}{N} \log \operatorname{det}\left(\mathbf{I}_{N M}+\gamma \mathbf{H}_{11} \mathbf{H}_{11}^{H} \mathbf{R}_{10}^{-1}\right),
\end{aligned}
$$

where

$$
\mathbf{R}_{01}=\mathbf{I}_{N M}+\gamma \mathbf{H}_{01} \mathbf{H}_{01}^{H} \text { and } \mathbf{R}_{10}=\mathbf{I}_{N M}+\gamma \mathbf{H}_{10} \mathbf{H}_{10}^{H} .
$$

\section{MMSE Linear Filtering per Polarization}

In this receiver architecture, each polarization is MMSEfiltered independently and the filtered signals are single-user decoded. The ergodic sum-rate capacity normalized by the number of cells is given by [17]:

$$
\begin{aligned}
\mathrm{C}_{4} \geq & -K \log \left(\frac{1}{N K} \operatorname{tr}\left[\left(\mathbf{I}_{N K}+\gamma \mathbf{H}_{00}^{H} \mathbf{R}_{01}^{-1} \mathbf{H}_{00}\right)^{-1}\right]\right) \\
& -K \log \left(\frac{1}{N K} \operatorname{tr}\left[\left(\mathbf{I}_{N K}+\gamma \mathbf{H}_{11}^{H} \mathbf{R}_{10}^{-1} \mathbf{H}_{11}\right)^{-1}\right]\right) .
\end{aligned}
$$

\section{E. Complexity}

It is known that the complexity for the optimal joint decoder is exponential [18] while for the MMSE is polynomial [19] with the channel matrix dimensions ${ }^{2}$. In a practical system, joint processing would be possible only within a cluster of BSs and therefore complexity would just depend on the number of streams per cell which have to be jointly processed. As a result, the complexity for the aforementioned joint decoders is: IV-A) exponential with $2 K$, IV-B) polynomial with $2 K$, IV-C) exponential with $K$, IV-D) polynomial with $K$.

\section{ERgodic CAPACITY ANALYSiS}

In this section, the ergodic sum-rate capacity analysis of the aforementioned receiver architectures is studied analytically. In order to produce tractable analytical expressions, an asymptotic regime is considered where the number of cells $N$ grows to infinity:

$$
\mathbb{C}=\lim _{N \rightarrow \infty} \mathbb{E}[\mathrm{C}] .
$$

\section{A. Optimal Joint Decoder}

Theorem 5.1: Using the optimal joint decoder, the per-cell ergodic sum-rate capacity converges almost surely (a.s) to:

$$
\mathbb{C}_{1} \stackrel{a . s}{\longrightarrow} 2 K \mathcal{V}_{\mathrm{MP}}\left((1+\chi) q(\boldsymbol{\Sigma}) N \gamma, \frac{K}{M}\right) .
$$

\footnotetext{
${ }^{2}$ it should be noted that $M \geq K$ is required for effective MMSE filtering.
}

Proof: Based on [4], the ergodic ergodic capacity can be expressed as:

$$
\mathbb{C}_{1} \stackrel{a . s}{\longrightarrow} 2 K \mathcal{V}_{\mathrm{MP}}\left(q(\boldsymbol{\Omega}) N \gamma, \frac{K}{M}\right)
$$

$$
\begin{aligned}
\text { where } & \mathcal{V}_{\mathrm{MP}}(u, v)=\log \left(1+u-\frac{1}{4} \phi(u, v)\right) \\
& +\frac{1}{v} \log \left(1+u v-\frac{1}{4} \phi(u, v)\right)-\frac{1}{4 u v} \phi(u, v), \\
\phi(u, v)= & \left(\sqrt{u(1+\sqrt{v})^{2}+1}-\sqrt{u(1-\sqrt{v})^{2}+1}\right)^{2} .
\end{aligned}
$$

The SNR scaling is given using the function $q$ which yields the Frobenius norm of matrix normalized by the number of elements in the matrix:

$$
\begin{aligned}
q(\boldsymbol{\Omega}) & =\frac{\left\|\left(\boldsymbol{\Sigma} \otimes \mathbb{I}_{2}\right) \odot\left(\mathbb{I}_{N M \times N K} \otimes \mathbf{X}\right)\right\|^{2}}{4 N^{2} K M} \\
& =q(\boldsymbol{\Sigma}) \frac{(1+\chi)}{2} .
\end{aligned}
$$

\section{B. MMSE Linear Filtering}

Theorem 5.2: Using MMSE linear filtering, the per-cell ergodic sum-rate capacity converges almost surely (a.s) to:

$$
\mathbb{C}_{2} \stackrel{a . s}{\longrightarrow}-2 K \log \left(\eta_{\mathrm{MP}}\left((1+\chi) q(\boldsymbol{\Sigma}) N \gamma, \frac{K}{M}\right)\right) .
$$

Proof: Based on [4], the ergodic sum-rate capacity can be expressed as:

$$
\mathbb{C}_{2} \stackrel{a . s}{\longrightarrow}-2 K \log \left(\eta_{\mathrm{MP}}\left(q(\boldsymbol{\Omega}) N \gamma, \frac{K}{M}\right)\right),
$$

where

$$
\eta_{\mathrm{MP}}(u, v)=1-\frac{\phi(u, v)}{4 u v}
$$

and $q$ given by eq. (15).

Lemma 5.1: An alternative expression can be derived using [20]:

$$
\begin{aligned}
& \mathbb{C}_{2}=2 K N \mathbb{C}_{1}-\left(4 K^{2} N-2 K\right) . \\
& \mathcal{V}_{\mathrm{MP}}\left((1+\chi) q(\tilde{\boldsymbol{\Sigma}})\left(N-\frac{1}{2 K}\right) \gamma, \frac{K-\frac{1}{2 N}}{M}\right),
\end{aligned}
$$

where $\tilde{\boldsymbol{\Sigma}}$ is obtained by $\boldsymbol{\Sigma}$ by removing a random column.

\section{Optimal Joint Decoder per Polarization}

Theorem 5.3: Using the optimal joint decoder per polarization, the per-cell ergodic sum-rate capacity converges almost surely (a.s) to:

$$
\begin{aligned}
\mathbb{C}_{3} \stackrel{a . s}{\longrightarrow} & 4 K \mathcal{V}_{\mathrm{MP}}\left((1+\chi) q(\boldsymbol{\Sigma}) N \gamma, \frac{2 K}{M}\right) \\
& -2 K \mathcal{V}_{\mathrm{MP}}\left(\chi q(\boldsymbol{\Sigma}) N \gamma, \frac{K}{M}\right) .
\end{aligned}
$$


TABLE I

PARAMETERS FOR CAPACITY RESULTS

\begin{tabular}{l|c|c|c|r} 
Parameter & & Value & Range & Figure \\
\hline Cell size & $D$ & $200 m$ & $0-1000 m$ & 2 \\
Inverse XPD factor & $\chi$ & 0.5 & $0-1$ & 3 \\
Number of BSs & $N$ & 5 & & \\
UTs per cell & $K$ & 4 & & \\
Dual Polar Ant. per BS & $M$ & 4 & & \\
UT Transmit SNR & $\gamma$ & $28.78 d B$ & & \\
Number of MC iterations & & $10^{3}$ & & \\
\hline
\end{tabular}

Proof: Since the two components of eq.(9) are statistically equivalent,

$$
\begin{aligned}
\mathbb{C}_{3}= & \frac{2}{N} \mathbb{E}\left[\log \operatorname{det}\left(\mathbf{I}_{N K}+\gamma \mathbf{H}_{00} \mathbf{H}_{00}^{H} \mathbf{R}_{01}^{-1}\right)\right] \\
= & \frac{2}{N} \mathbb{E}\left[\log \operatorname{det}\left(\mathbf{I}_{N K}+\gamma \mathbf{H}_{0} \mathbf{H}_{0}^{H}\right)\right] \\
& -\frac{2}{N} \mathbb{E}\left[\log \operatorname{det}\left(\mathbf{I}_{N K}+\gamma \mathbf{H}_{01} \mathbf{H}_{01}^{H}\right)\right] .
\end{aligned}
$$

Using similar arguments as in the proof of theorem 5.1, eq. (21) yields eq. (20).

\section{MMSE Linear Filtering per Polarization}

Lemma 5.2: Using MMSE linear filtering per polarization, the per-cell ergodic sum-rate capacity is given by:

$$
\begin{aligned}
& \mathbb{C}_{4}=2 K N\left(\mathbb{C}_{3}-\tilde{\mathbb{C}}_{3}\right) \text { with } \tilde{\mathbb{C}}_{3}= \\
& \left(4 K-\frac{2}{N}\right) \mathcal{V}_{\mathrm{MP}}\left((1+\chi) q(\tilde{\boldsymbol{\Sigma}})\left(N-\frac{1}{2 K}\right) \gamma, \frac{2 K-\frac{1}{N}}{M}\right) \\
& -K \mathcal{V}_{\mathrm{MP}}\left(\chi q(\boldsymbol{\Sigma}) N \gamma, \frac{K}{M}\right) .
\end{aligned}
$$

Proof: Since the two components of eq. (11) are statistically equivalent,

$$
\mathrm{C}_{4}=-2 K \mathbb{E}\left[\log \left(\frac{1}{N K} \operatorname{tr}\left[\left(\mathbf{I}_{N K}+\gamma \mathbf{H}_{00}^{H} \mathbf{R}_{01}^{-1} \mathbf{H}_{00}\right)^{-1}\right]\right)\right] .
$$

The expression for $\mathbb{C}_{4}$ can be derived using [20].

\section{NuMERICAL RESUlts}

In the present section, a number of numerical results is presented in order to evaluate the system's ergodic sum-rate capacity under the proposed receiver architectures and verify the accuracy of the derived analytical expressions. System capacity is calculated for a typical $X P D$ factor 2 and variable cell size, as well as for variable $X P D$ and a typical cell size of $200 \mathrm{~m}$. More specifically, simulations are performed by generating $10^{3}$ instances of random Gaussian matrices, each one representing a single fading realization of the system. In addition, the user variance profile matrices are constructed deterministically according to the path-loss model in [4] and used to shape the variance of the i.i.d c.s.s. elements ${ }^{3}$. Subsequently, the per-cell ergodic capacities are evaluated using: a)

\footnotetext{
${ }^{3}$ It should be noted that the presented analysis is generic and can be used for any path-loss function or cellular array model (linear, hexagonal etc.)
}

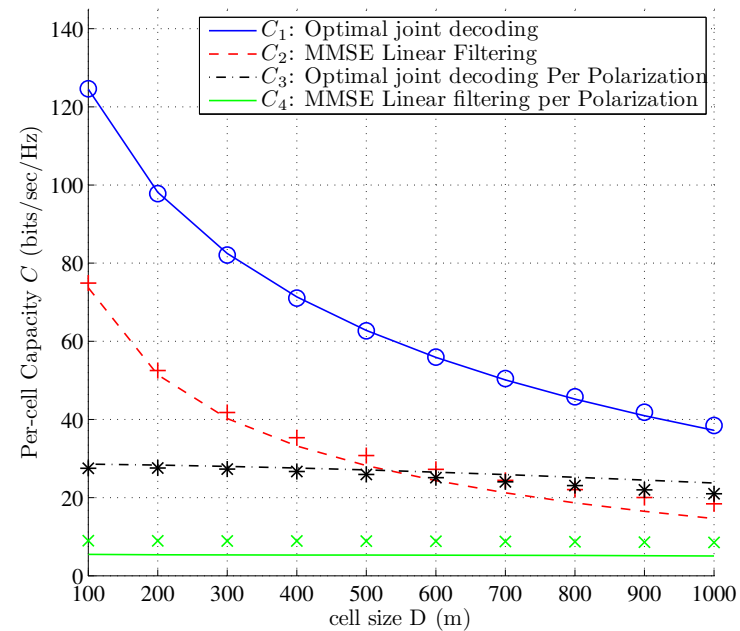

Fig. 2. Per-cell ergodic sum-rate capacity scaling versus the cell size $D(m)$.

eq.(7) for the optimal joint decoder b) eq. (8) for the MMSE Linear filtering architecture c) eq. (9) for MMSE joint decoder per polarization and d) eq. (11) for the MMSE linear filtering per polarization receiver architecture. The capacity for each architecture is averaged over all iterations and plotted versus cell size (fig. 2) and versus the inverse XPD factor $\chi$ (fig.3). On the same figures, analytical curves resulting from equations a)eq. (13) b) Lemma (5.1) c) eq. (20) d) eq. (22) ${ }^{4}$ for each receiver architecture respectively, are opposed to the simulation results. In the aforementioned figures, points represent values calculated through Monte Carlo simulations, while lines refer to curves resulting from the analytical expressions of section V. Finally, table I presents an overview of the parameter values and ranges used for producing the numerical results.

The decrease of capacity as the cell size increases is perceptible in figure 2. This results from the decrease of the received power at the BSs since the uniformly distributed users move away from the BS as cells expand. Moreover, optimal capacity is achieved by jointly decoding received signals from both polarizations $\left(C_{1}\right)$. Sub-optimal less complex receiver architectures tend to approach the optimal capacity limit in the sparse cellular regime $(D=1000 m)$. An important observation is that for small cell sizes $(D<400 m)$ MMSE Linear Filtering of both polarizations $\left(C_{2}\right)$ achieves remarkably higher capacity than the optimal decoding of each polarization separately $\left(C_{3}\right)$. However, the latter case does not apply for large cell sizes $(D>600 m)$. Finally, it can be seen that per polarization processing $\left(C_{3}, C_{4}\right)$ is less sensitive to the cell size, since the performance is mainly dictated by the received cross-polar interference.

Figure 3 depicts how the variation of the cross polar discrimination affects the system performance, for each receiver architecture. When both polarizations are jointly decoded $\left(C_{1}, C_{2}\right)$,

\footnotetext{
${ }^{4}$ Eq. (5.1) seems to be less accurate than the rest because it includes a multiplicative term $(2 K N)$ which scales with the channel matrix dimensions and intensifies the deviation of the term $\mathbb{C}_{3}-\widetilde{\mathbb{C}}_{3}$.
} 


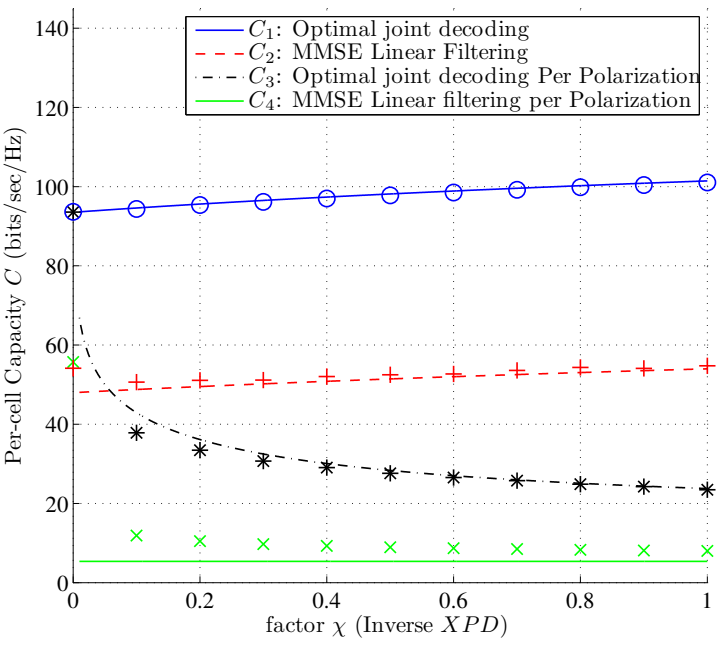

Fig. 3. Per-cell ergodic sum-rate capacity scaling versus factor $\chi$ (inverse $X P D)$.

capacity increases as factor $\chi$ increases (or alternatively as $X P D$ decreases). Since both co- and cross-polar components are jointly processed, less cross polar discrimination leads to higher capacities, due to higher received power. On the other hand, when polarizations are treated separately, the increase of $\chi$ (decrease of $X P D$ ) results in increased cross polar interference, thus lower system capacities. Subsequently, it can be concluded that decoding per polarization is favoured by high $X P D$ factors. However, systems with poor discrimination between polarizations should exploit the cross-polar component in order to achieve higher ergodic capacity.

\section{CONCLUSIONS \& FUTURE WORK}

Dual polarization has been traditionally employed in order to save hardware space or combat channel impairments, such as Faraday rotation. The main design objective was to create parallel channels with low correlation and high power imbalance, which favour individual processing per polarization. In this paper, it is shown that joint polarization processing is beneficial for MJD, especially in low-XPD dense systems. Per polarization processing could be employed in high-XPD enviroments in order to reduce the complexity of the joint receiver.

An immediate extension of the work carried out so far is the incorporation of correlation between the dual polarized antennas at every user and at the base station as well as the correlation between collocated antenna pairs at the base station. This could be achieved using the well known Kronecker model. Additionally, future work includes the investigation of Rician dual-polar channels. Specifically, the comparison between highly-correlated co-polar antenna versus lowly-correlated dual-polar antenna systems, both using all the MJD techniques would be of utmost importance.

\section{ACKNOWLEDGMENT}

This work was partially supported by the National Research Fund, Luxembourg.

\section{REFERENCES}

[1] D.-S. Shiu, G. Foschini, and M. G. J. Kahn, "Fading correlation and its effect on the capacity of multielement antenna systems," IEEE Trans. Commun., vol. 48, no. 3, pp. 502-513, Mar 2000.

[2] G. J. Foschini and M. J. Gans, "On limits of wireless communications in a fading environment when using multiple antennas," Wirel. Pers. Commun., vol. 6, no. 3, pp. 311-335, 1998.

[3] 3GPP, "Further advancements for E-UTRA physical layer aspects," 3rd Generation Partnership Project, Tech. Rep. TR 36.814.

[4] S. Chatzinotas, M. Imran, and R. Hoshyar, "On the multicell processing capacity of the cellular MIMO uplink channel in correlated Rayleigh fading environment," IEEE Trans. Wireless Commun., vol. 8, no. 7, pp. 3704-3715, July 2009

[5] S. Chatzinotas, M. Imran, and C. Tzaras, "On the capacity of variable density cellular systems under multicell decoding," IEEE Commun. Lett., vol. 12, no. 7, pp. 496 - 498, Jul 2008.

[6] D. Kaltakis, M. Imran, and C. Tzaras, "Information theoretic capacity of cellular multiple access channel with shadow fading," Communications, IEEE Transactions on, vol. 58, no. 5, pp. 1468 -1476, May 2010.

[7] O. Somekh and S. Shamai, "Shannon-theoretic approach to a Gaussian cellular multiple-access channel with fading," IEEE Trans. Inf. Theory, vol. 46, no. 4, pp. 1401-1425, Jul 2000.

[8] P. Horvath and I. Frigyes, "Application of the 3D polarization concept in satellite MIMO systems," in Global Telecommunications Conference, 2006. GLOBECOM '06. IEEE, Dec 2006, pp. 1 -5.

[9] J. F. Valenzuela-Valdes, M. A. Garcia-Fernandez, A. M. MartinezGonzalez, and D. Sanchez-Hernandez, "The role of polarization diversity for MIMO systems under rayleigh-fading environments," Antennas and Wireless Propagation Letters, IEEE, vol. 5, no. 1, pp. 534 -536, 2006.

[10] P. Kyritsi, D. Cox, R. Valenzuela, and P. Wolniansky, "Effect of antenna polarization on the capacity of a multiple element system in an indoor environment," Selected Areas in Communications, IEEE Journal on, vol. 20, no. 6, pp. 1227 - 1239, Aug. 2002.

[11] H. Bolcskei, R. Nabar, V. Erceg, D. Gesbert, and A. Paulraj, "Performance of spatial multiplexing in the presence of polarization diversity," in Acoustics, Speech, and Signal Processing, 2001. Proceedings. (ICASSP '01). 2001 IEEE International Conference on, vol. 4, 2001, pp. $2437-2440$.

[12] C. Degen and W. Keusgen, "Performance of polarisation multiplexing in mobile radio systems," Electronics Letters, vol. 38, no. 25, pp. 1730 - 1732, Dec. 2002.

[13] A. Tulino, A. Lozano, and S. Verdú, "Impact of antenna correlation on the capacity of multiantenna channels," IEEE Trans. Inf. Theory, vol. 51, no. 7, pp. 2491-2509, Jul 2005.

[14] A. Tulino, S. Verdu, and A. Lozano, "Capacity of antenna arrays with space, polarization and pattern diversity," in Information Theory Workshop, 2003. Proceedings. 2003 IEEE, 31 March-4 April 2003, pp. 324-327.

[15] W. Hachem, P. Loubaton, and J. Najim, "Deterministic equivalents for certain functionals of large random matrices," Annals of Applied Probability, vol. 17, pp. 875-930, 2007. [Online]. Available: http://arxiv.org/abs/math.PR/0507172

[16] M. K. Varanasi and T. Guess, "Optimum decision feedback multiuser equalization with successive decoding achieves the total capacity of the Gaussian multiple-access," in 31st Asilomar Conference on Signals, Systems and Computers (ACSSC' 97), vol. 2, Nov 1997, pp. 1405-1409.

[17] B. Li, Q. Wang, G. Lu, Y. Chang, and D. Yang, "Linear MMSE frequency domain equalization with colored noise," in Vehicular Technology Conference, 2007. VTC-2007 Fall. 2007 IEEE 66th, Oct. 2007, pp. $1152-1156$.

[18] S. Verdú, Multiuser Detection. UK: Cambridge University Press, 1998

[19] E. Biglieri, R. Calderbank, A. Constantinides, A. Goldsmith, A. Paulraj, and H. V. Poor, MIMO Wireless Communications. New York, NY, USA: Cambridge University Press, 2007.

[20] M. McKay, I. Collings, and A. Tulino, "Achievable sum rate of MIMO MMSE receivers: A general analytic framework," IEEE Trans. Inf Theory, vol. 56, no. 1, pp. $396-410$, Jan. 2010. 